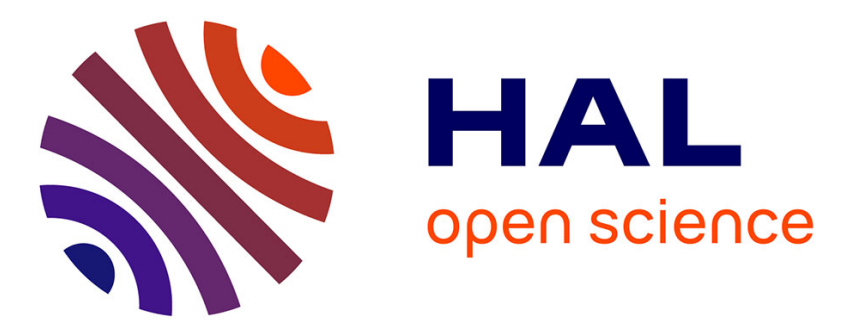

\title{
Femtosecond responses of hydrated DNA irradiated by ionizing rays focus on the sugar-phosphate part
}

\author{
Aurélien de La Lande
}

\section{To cite this version:}

Aurélien de La Lande. Femtosecond responses of hydrated DNA irradiated by ionizing rays focus on the sugar-phosphate part. Theoretical Chemistry Accounts: Theory, Computation, and Modeling, 2021, 140 (7), pp.77. 10.1007/s00214-021-02778-1 . hal-03456033

\section{HAL Id: hal-03456033 https://hal.science/hal-03456033}

Submitted on 30 Nov 2021

HAL is a multi-disciplinary open access archive for the deposit and dissemination of scientific research documents, whether they are published or not. The documents may come from teaching and research institutions in France or abroad, or from public or private research centers.
L'archive ouverte pluridisciplinaire HAL, est destinée au dépôt et à la diffusion de documents scientifiques de niveau recherche, publiés ou non, émanant des établissements d'enseignement et de recherche français ou étrangers, des laboratoires publics ou privés. 


\title{
Femtosecond Responses of Hydrated DNA Irradiated by lonizing Rays, Focus on the Sugar-Phosphate Part
}

\author{
Aurélien de la Lande ${ }^{1}$ \\ 1 : Institut de Chimie Physique, Université Paris Saclay, CNRS, (UMR 8000). France. \\ To whom correspondence should be addressed: aurelien.de-la-lande@universite-paris-saclay.fr
}

\begin{abstract}
In this article we investigate the mechanisms of DNA ionization upon irradiation by $0.5 \mathrm{MeV}$ alpha particles. We focus on the sugar-phosphate group and its hydration shell. In radiation chemistry, the term quasi-direct effect refers the physical and chemical responses taking place after irradiation of solvent molecules pertaining to the solvation shells of solutes. The molecular mechanisms accounting for the quasi-direct effect are actually largely elusive, especially for those prevailing in the early time scales $\left(<10^{-12} \mathrm{~s}\right)$. We report Real-Time-Time-Dependent Auxiliary Density Functional Theory simulations carried out within the framework of hybrid QM/MM scheme (Quantum Mechanics/Molecular Mechanics) with polarizable and non-polarizable embedding. Ten water molecules from the solvation shell of DNA backbone are independently irradiated. We find that during the first femtoseconds after irradiation, the holes formed on the irradiated water remains at their sites of formation. Electrostatic induction within the environment doesn't significantly impact charge migrations. We address the hypothesis that charge migration driven by electron correlation is responsible for an ultrafast $\mathrm{H}_{2} \mathrm{O}^{+}$-toDNA charge transfers, which would account for a quasi-direct effect. We find that pure charge migration at fixed nuclear positions is not responsible for the quasi-direct effect when considering sugar-phosphate solvation shells.
\end{abstract}




\section{Introduction}

Chemical damages induced by ionizing radiations on biological molecules like DNA or proteins are customarily classified as stemming either from direct[1] or indirect effect[2]. The former refers to lesions triggered by direct energy deposition into the biomolecules upon interaction with ionizing radiation. The latter, on the other hand, refers to chemical alterations involving the products of water radiolysis, notably the $\mathrm{OH} \bullet$ or the $\mathrm{H} \bullet$ radicals, that damage, after diffusion, chemical functions on biomolecules. The respective importance of one effect over the other is still debated[3], but both undoubtedly contribute to cellular metabolism malfunctions. In fact, the relative importance of one effect over the other is likely to be dependent on the hydrometric content of cellular compartments[4].

In bulk water $\mathrm{H}_{2} \mathrm{O}^{+}$gives a proton to a solvent molecule in less than $100 \mathrm{fs}$, as suggested from the early work of Gauduel et al[5]. Recently, Loh et al. reported a value of $46 \pm 10 \mathrm{fs}$ from pump-probe measurements with a tunable femtosecond soft $x$-ray pulses from an X-ray free electron laser[6]. Such a time scale is in line with numerical simulations, for instance those reported by Loh et al.[6] or by Marsalek et al.[7]

Besides the direct and indirect effects, a so-called quasi-direct effect has also been put forward many years ago by Sevilla and co-workers to account for intriguing experimental results obtained upon $\gamma$ irradiation of glassy DNA samples at low temperature and various hydration levels $[8,9]$. The quasidirect effect refers to damages triggered by the primary irradiation of the solvation water molecule that react directly with DNA. For example, the $\mathrm{H}_{2} \mathrm{O}^{+}$cation can oxidize DNA before transferring a proton to a surrounding molecule, as normally the case for bulk water.

In the experiments reported by Sevilla and co-workers, the temperature was maintained at 77K to slow down proton transfer reactions and to detect the signature of radical species on longer time by Electron Paramagnetic Resonance. But cleary, at room temperature electron and proton transfer probably compete within the hydration shell of DNA. Over the last decade, Mostafavi and co-workers deployed an intensive research program relying on picosecond pulse radiolysis of highly concentrated 
solutions (e.g., sulfuric, phosphoric, nitric acids solutions, to characterize the redox properties of $\mathrm{H}_{2} \mathrm{O}^{+}[10-13]$. More recently they obtained evidences of ultrafast hole transfer from $\mathrm{H}_{2} \mathrm{O}^{+}$to nucleosides and nucleotides with the same technology $[14,15]$. It was also showed by simulations that $\mathrm{H}_{2} \mathrm{O}^{+}$can catalyze the hydroxylation of a nucleobase without proceeding through the formation of the HO^ radical [16]. The quasi-direct effect is now incorporated in the database of physical and physicalchemical events available Monte-Carlo track structure codes[17].

The precise molecular mechanisms responsible for the quasi-direct effect are actually not completely clear. In particular the precise charge transfer mechanism from $\mathrm{H}_{2} \mathrm{O}^{+}$to DNA remain elusive[15]. An electron transfer following the canonical Marcus Theory is a possibility, but other processes such as femtosecond charge migrations are also plausible and the objective of the present work is to assess this eventuality.

The terminology "charge migration" refers to a very specific charge transfer mechanism put forward by Cederbaum and co-workers in the field of attosecond sciences[18, 19]. Contrary to the canonical electron transfer chemical reactions that are driven by nuclear motions (see for instance the Marcus theory[20]), charge migrations are propelled by electron correlations, by definition at (almost) fixed nuclear positions. This phenomenon arises when an electronic system is driven away from its equilibration situation, that is after a more or less strong perturbation. The characteristic time scales of charge migration range from a few tens of attoseconds to a few femtoseconds, supporting the idea that charge migrations are largely decoupled from, though interconnected with, nuclear motion. In the experiments developed in the group of Prof. Sevilla, the samples were brought for the needs of the experiment to a temperature of $77 \mathrm{~K}$, at which nuclear motions are expected to be largely frozen, hence suggesting a charge migration mechanism might be at play.

Another result supporting this idea comes from a recent experimental/computational study of the radiolysis of sulfuric acid solutions[21]. We showed from computer simulations that ultrafast charge transfer between $\mathrm{H}_{2} \mathrm{O}^{+}$and sulfuric acid was feasible in the sub-picosecond time domain by charge 
migration, while nuclear-motion-driven electron transfer was not competitive on this time-scale. This mechanistic proposal offered a plausible explanation of the ps pulse radiolysis results.

Here, we rely on electron dynamics simulations carried out in the framework of Real-Time TimeDependent Auxiliary Density Functional Theory[22, 23] (RT-TD-ADFT) coupled to molecular mechanics to investigate DNA irradiation by alpha particle and subsequent charge migrations within the hydrated sugar-phosphate moieties. We compare two QM/MM implementations, relying either on the AMBER ff99[24] force field or on the polarizable ff02[25] force field that includes electrostatic induction trough the charge point dipole model. We first describe the methodology before investigating irradiation of the solvation water molecules and the phosphate groups.

\section{Methodology}

All simulations have been carried out with a developer version of deMon2k [26] (version 4.4.5), which is readily available on request to the author. Our approach implements a hybrid QM/MM methodology coupling Real-Time Time-Dependent Auxiliary Density Functional Theory (RT-TD-ADFT) to Molecular Mechanics[27, 28]. The QM engine relies on Auxiliary DFT that complies to the Kohn-Sham framework but makes use of variationally fitted electron densities (i.e. auxiliary electron densities) to evaluate classical electron repulsion terms and exchange-correlation (XC) matrix elements[29].

The initial structure was taken from Ref.[30]. The simulated system consists of a DNA oligomer, the sequence of which is AAAGAAA/TTTCTTT, solvated in a $60 \AA$ diameter water droplet and $12 \mathrm{Na}^{+}$cations to ensure electrical neutrality. The system has been equilibrated by classical molecular dynamics simulations and then optimized at the MM level with the Amber package[31, 32]. We then moved to $\mathrm{QM} / \mathrm{MM}$ simulation with deMon2k. To this end the full system was pruned to fit within a sphere keeping all molecules situated within $30 \AA$ from the center-of-mass of the QM region. For the present study we have selected as QM region the sugar-phosphate moieties of a thymine base and the closest 26 water molecules (Figure 1), for a total of 96 atoms and 346 electrons. The charge of the QM region 
was set to -1 . The rest of the system is described at the MM level $\left(9,607\right.$ atoms) and contains $8 \mathrm{Na}^{+}$ cations that lie outside of the QM region.

RT-TD-ADFT simulations are launched from the stationary solution of the time-independent KohnSham equations obtained by the Self-Consistent-Field (SCF) procedure with tight convergence criteria $\left(10^{-10} \mathrm{Ha}\right.$ and $10^{-7} \mathrm{Ha}$ for the MinMax SCF energy[33] and for the auxiliary density fitting energy convergence threshold respectively). We have used the PBE exchange correlation (XC) functional[34] with the combination of AUG-CC-pVDZ[35] and GEN-A2* atomic orbital and auxiliary basis sets to expand the Kohn-Sham Molecular Orbitals (MO) and the auxiliary electronic densities respectively. The XC contributions have been calculated by numerical integration over Lebedev grids, adapted to provide an accuracy of $10^{-7} \mathrm{Ha}$ on the diagonal elements of the XC matrix[36]. The electronic equation-ofmotions have been solved with the second order Magnus propagator[37] coupled to a predictorcorrector scheme[38], with a time step of $0.001 \mathrm{fs}$. The exponential of the Kohn-Sham matrix has been evaluated with a Taylor expansion truncated at the $45^{\text {th }}$ order evaluated with the Scalapack library [39]. The chosen simulation parameters ensure energy conservation up to $10^{-9} \mathrm{Ha}$ during RT-TD-ADFT simulations. The Kohn-Sham Hamiltonian has been supplemented with a Complex Absorbing Potential $\left(V_{C A P}\right)$ defined over the real space. $V_{C A P}$ is defined from a superposition of atom-centered CAP functions: $V_{C A P}(R)=\min _{a} V_{C A P}^{a}(R)$, with:

$V_{C A P}^{a}(R)= \begin{cases}0 & \text { for } R<R^{\circ} \\ V^{\max } \sin ^{2}\left(\frac{\pi}{2 W}\left(R-R^{\circ}\right)\right) \text { for } & R^{\circ}+W<R<R^{\circ} \\ V^{\max } & R>R^{\circ}+W\end{cases}$

where $V^{\max }$ is the maximum value of the absorbing potential, $W$ is the width of increase of the atomic CAP and $R^{\circ}$ is the distance threshold at which starts the atomic CAP. $V^{\max }, R^{\circ}$ and $W$ were set to 10 Ha, $7 \AA$ and $15 \AA$ respectively. The matrix elements of the $V_{C A P}$ have been evaluated by numerical integration over Lebedev grids encompassing 99 radial shells and 590 angular points per shells and the partitioning scheme introduced by Becke[40]. 
We use an in-house implementation of additive $Q M / M M$, the description of which can be found in [27, 41]. Three link atoms have been introduced to saturate the valence of the atoms lying at the $Q M / M M$ frontier. We have used both a polarizable and a non-polarizable version of the AMBER force field[25]. Polarizable simulations ( $\mathrm{QM} / \mathrm{MMpol}$ ) are afforded by the point-charge dipole model of electrostatic induction. Namely, an induced dipole is calculated on each $\mathrm{MM}$ atom as the product of its polarizability, that we assume to be isotropic, and the electric field at the atom site. The electric field encompasses the electric field generated by other MM atoms (permanent charges and induced dipoles) and by the QM region. For computational efficiency, the latter is evaluated from the auxiliary electron density. We previously showed that with the use of the GEN-A2* auxiliary basis set, this approximation yields accurate results[28]. In QM/MMpol simulations, the MM environment is polarized on-the-fly by the non-stationary electron density on the QM region, while the electron cloud feels the back reaction of the environment via the potential created by the MM induced dipoles which is incorporated into the electronic Kohn Sham potential. We make the reasonable approximation that the MM induced dipoles have time to fully relax between two RT-TD-ADFT propagation steps. An iterative solver is used to determine induced dipoles. The iterations are pursued until the Root-MeanSquare of induced dipoles lies below $10^{-10} \mathrm{D}$. To avoid over-polarization troubles, the electric fields generated by the MM dipoles are damped with the Thole formulas [42, 43]. Also, the polarizabilities of $\mathrm{MM}$ atoms directly connected to the $\mathrm{MM}$ atom of the $\mathrm{QM} / \mathrm{MM}$ border have been set to zero. 

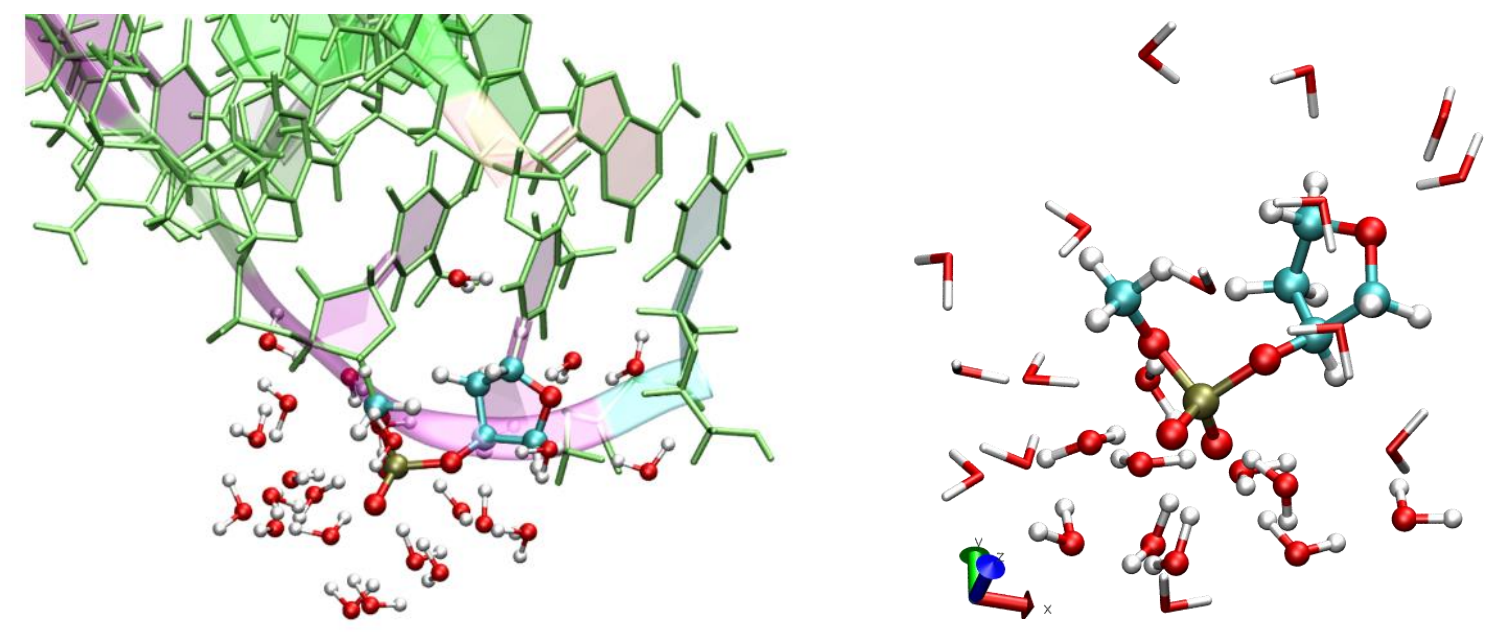

Figure 1: Left, QM/MM simulation set-up highlighting the QM region with ball-and-stick representation (color code: $O$ in red, $C$ in cyan, $H$ in white and $P$ in gold). Water molecules and $\mathrm{Na}^{+}$counter-ions described at the MM level are not shown for clarity. Right, zoom on the QM region

The projectile, a $\mathrm{He}^{2+}$ nucleus, was positioned $30 \AA$ away from the center of mass of the QM region at the beginning of the RT-TD-ADFT simulations, defining by convention time zero. The projectile was assigned a propagation line and a kinetic energy $(0.5 \mathrm{MeV})$, that have been maintained throughout the simulation. We thus consider in this work inelastic collisions between charged particles and matter. Note anyway, that for such high kinetic energies of the projectile, the inelastic channel is dominant over elastic ones. We have separately targeted ten water molecules located in the close environment of the phosphate oxygen atoms, defining a propagation line going through the center of mass of the targeted water molecule.

During RT-TD-ADFT simulations, partial atomic charges have been calculated every 10 as according to the Hirshfeld population scheme $[44,45]$. To reduce the computational cost of repetitive charge analyses, this was achieved by numerically integrating the auxiliary electron density instead of the Kohn-Sham one. Our tests showed this substitution is safe when using the flexible GEN-A2* auxiliary basis set[45]. 
All the data produced by deMon $2 k$ have been analyzed with the $R$ package for statistical computing[46]. The reader can find these data in supplementary material as well as all the scripts written to produce the graphs and the tables of this article.

\section{Results}

We have run and analyzed two campaigns of RT-TD-ADFT simulations, in which the $\mathrm{He}^{2+}$ ion is targeting either the phosphate group or its solvation shell. We start the description of our results with the latter.

\subsection{Solvation shell irradiation}

Ten collision conditions have been considered, each one targeting one water molecule, but not the sugar-phosphate group. Figure 2 depicts the energy profiles during the collisional process and few femtoseconds after it. Graphs on the left-hand-side and right-hand-side have been obtained with nonpolarizable and polarizable embedding of the QM region, respectively. For these graphs, we have considered all energy contributions except the interaction energy between the projectile and the molecular system. We have subtracted the ground state energy so that all curves start at zero. Energy deposition is a very fast process that takes place when the projectile enters the electrons cloud of the QM region. This is seen from the very sharp variations of the energy around 580 as. Note that with our $\mathrm{QM} / \mathrm{MM}$ methodology, no energy is deposited into the MM region as by definition we don't describe the electronic structure explicitly in this region. The amount of energy deposited into the molecular system is huge and reaches almost $350 \mathrm{eV}$ in average. Such very high electronic excitations are hallmarks of radiation chemistry processes. The inclusion of induction within the MM region and within the QM/MM interaction doesn't dramatically modifies the picture. After collision, when the projectile is sufficiently far away from the molecular system, it doesn't interact with the molecular system and the total energy is nearly constant. The energy is not strictly constant because of the presence of the absorbing potential. However, we note that only a very small fraction of electron density is effectively absorbed by the CAP that lies $7 \AA$ away from the QM region (less than 0.01 e- are absorbed after $6 \mathrm{fs}$ ). 
The electrostatic potential created by the MM atoms acts as a confining potential, preventing highly excited electrons to escape the QM region.
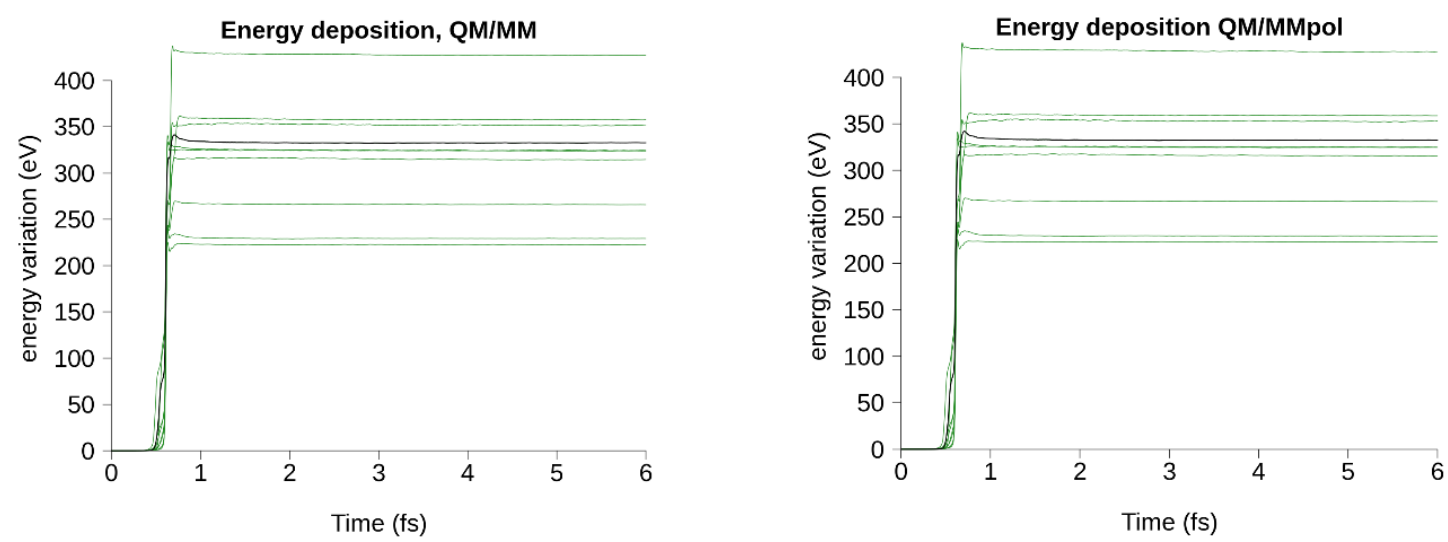

Figure 2: Energy profile upon collision of the QM region with a He ${ }^{2+}$ projectile for QM/MM (left) and QM/MMpol (right) simulations. We take the ground state energy as reference. The ten curves corresponds to ionization of a different water molecule. The black curves are the averages.

To investigate more in-depth energy deposition in RT-TD-ADFT/MMpol simulations we have plotted in Figure 3 the energy variations for the QM region, i.e. the sum of the electronic kinetic energy, of the classical Coulomb repulsion energy, of the XC energy and of the electron QM atom nuclei energy (Top), for the embedding energy by MM atoms punctual charges (Middle) and for the induction energy (Bottom). It is clear that the main contribution to overall energy deposition is, by far, the energy of the QM region. This result is in line with the local nature of electronic excitation. The embedding energy arising from to permanent point charges positioned on the $\mathrm{MM}$ atoms increases by a few electronvolts while the induction energy decreases by even a smaller amount. 

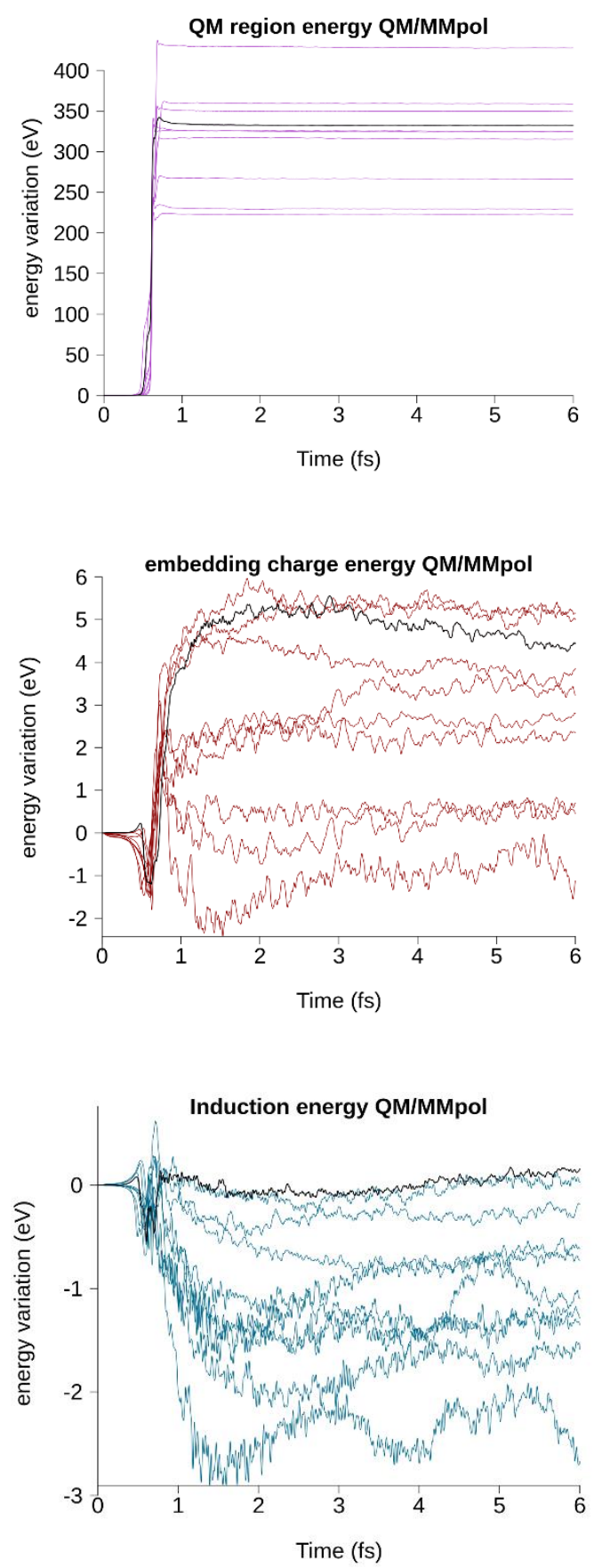

Figure 3: QM/MM energy contributions for the ten trajectories. The black curves are the average over the ten trajectories.

We now analyze the electronic dynamics taking place in the molecular system during and after collision with the alpha particle. To this end, we depict in Figure 4 the evolution of the fragment charges with respect to time for the water molecule stroke by the projectile (top), for the phosphate-sugar moieties (middle), and for the remaining water molecules (bottom). In each panel, the black curve is the average 
over the ten trajectories. Collision occurs around $0.58 \mathrm{fs}$ after the beginning of the simulation, as seen from the sharp variation of the molecular charges, in line with the energy variation described above. Depending on the simulation, the water molecule stroke by the projectile gains a charge comprised between 1 and 1.8. The curves then exhibit fast fluctuations on the attosecond timescale and a slower decay on the femtosecond timescale. The formers are signatures of charge migrations between the irradiated water and the surrounding molecules. The latter, on the other hand, indicates partial charge recombination. The electrons emitted upon ionization are scattered over the sugar-phosphate moieties and over the other water molecules, as deduced from the charges dropping below zero after ionization. In average, the charge on the sugar-phosphate moieties decreases from 0 to $-0.5 \mathrm{e}^{-}$before raising-up to $-0.2 \mathrm{e}^{-}$within $5 \mathrm{fs}$. On the other hand, the charge on the remaining water molecules remain in average constant over time. Each curve taken individually exhibits fast fluctuations on the sub-femtosecond time scale, that reflects fast charge migrations, but the slower femtosecond evolution suggests that charge recombination essentially takes place between the ionized water molecule and the sugar phosphate moieties. We show in Figure 5 the isosurface deformation density after collision for one of the trajectories. The orange isosurfaces show region of depletion of electronic density with respect to the ground state. Two water molecule below the phosphate group largely loose electron density already at $0.8 \mathrm{fs}$. We see that the deformation density doesn't extend much beyond the molecules stroke at the begining. Contribution from the sugar-phosphate group of from other water molecules are modest even a few femtoseconds after irradiation.

From a technical point of view, the inclusion of induction doesn't change these conclusions. It is apparent comparing in Figure 4 the right-hand-side graphs to the left-hand-side ones, that induction only marginally modifies charge dynamics. On the time scale considered here, we do not see during these simulations a net electron transfer from the phosphate-sugar to the $\mathrm{H}_{2} \mathrm{O}^{+}$that would sign a quasidirect effect (DNA $\left.+\mathrm{H}_{2} \mathrm{O}^{+} \rightarrow \mathrm{DNA}^{+}+\mathrm{H}_{2} \mathrm{O}\right)$ 

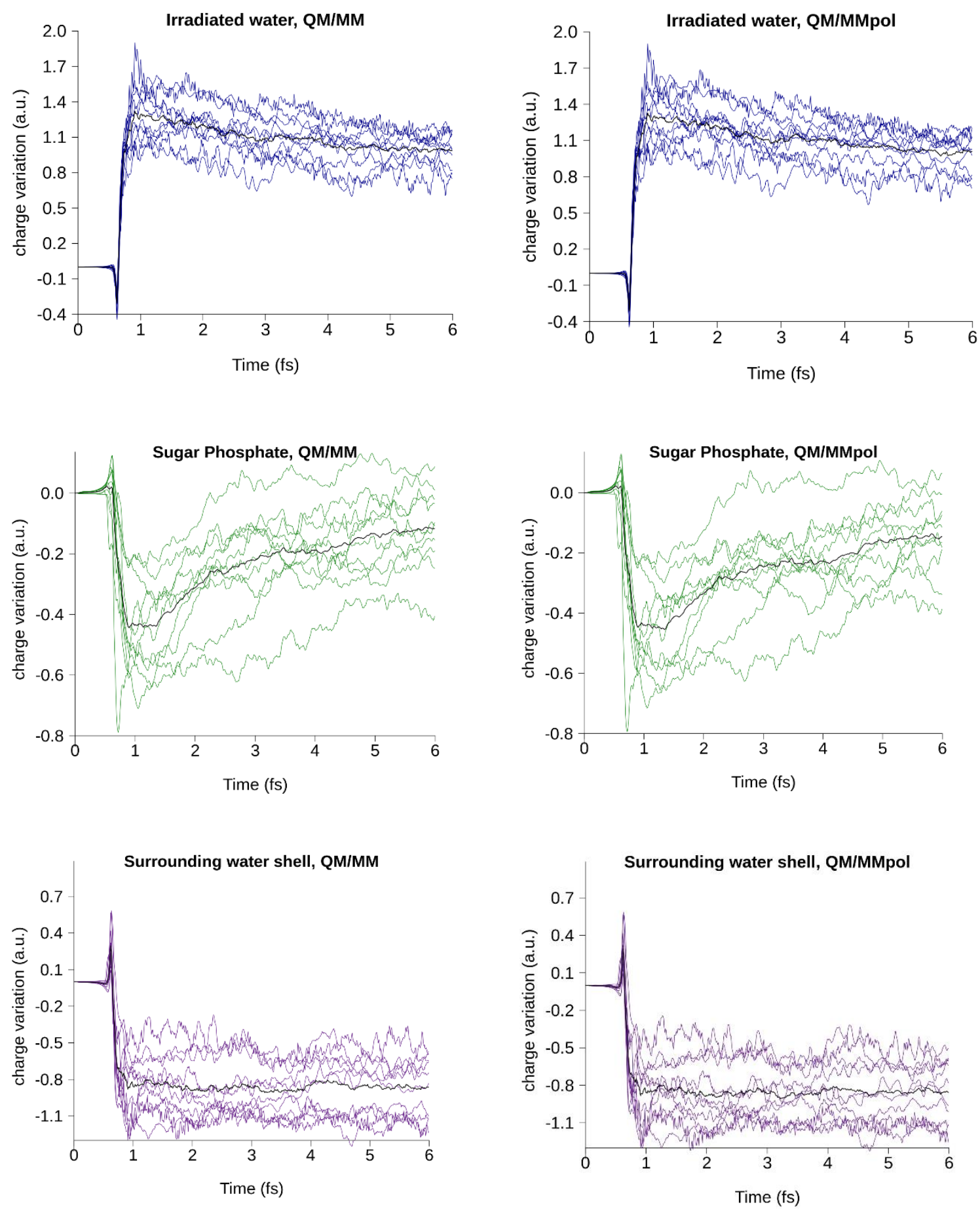

Figure 4: Evolution of the fragment charges with respect to the ground state following irradiation of a solvation water molecule by a $0.5 \mathrm{He}^{2+}$ ion. The simulations have been repeated targeting nine distinct was molecules by the projectile. The black curves are the average. 


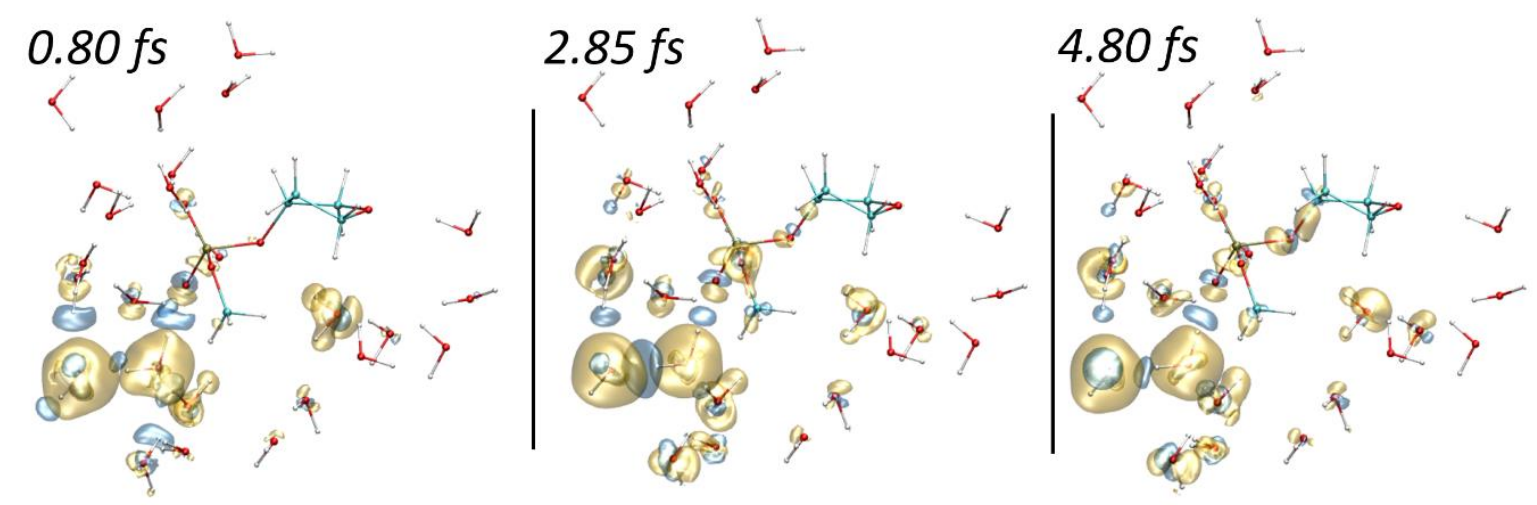

Figure 5: Isosurfaces (0.01 bohr $\left.{ }^{3}\right)$ of the deformation density with respect to the ground state at $0.8,2.85$ and $4.8 \mathrm{fs}$ after the beginning of the RT-TD-ADFT/MM simulations (collision takes place at $0.58 \mathrm{fs}$ ). Accumulation of density in blue and depletion of density in orange. Only the QM region is shown for clarity.

\subsection{Irradiation of the phosphate moieties.}

We now consider simulations in which the $\mathrm{He}^{2+}$ projectile targets the center-of-mass of the phosphate group $\left(\mathrm{O}_{3} \mathrm{PCH}_{2}-\right)$. Figure 6 depicts charge variation of the water shell, of the deoxyribose and of the phosphate group during $12 \mathrm{fs}$ with a collision time taking place around $0.6 \mathrm{fs}$. Three collision trajectories have been considered in which the projectile travels along the $\mathrm{x}, \mathrm{y}$ or $\mathrm{z}$ axis (see Figure 1 ). Following the conclusions of the previous section we have only used a non-polarizable $\mathrm{QM} / \mathrm{MM}$ approach here.

As expected the collision triggers the formation of holes on the sugar-phosphate moieties with emission of electron within the water solvation shell. During the $11 \mathrm{fs}$ following ionization we observe very fast charge fluctuations but also a rather steady and slow charge recombination. 

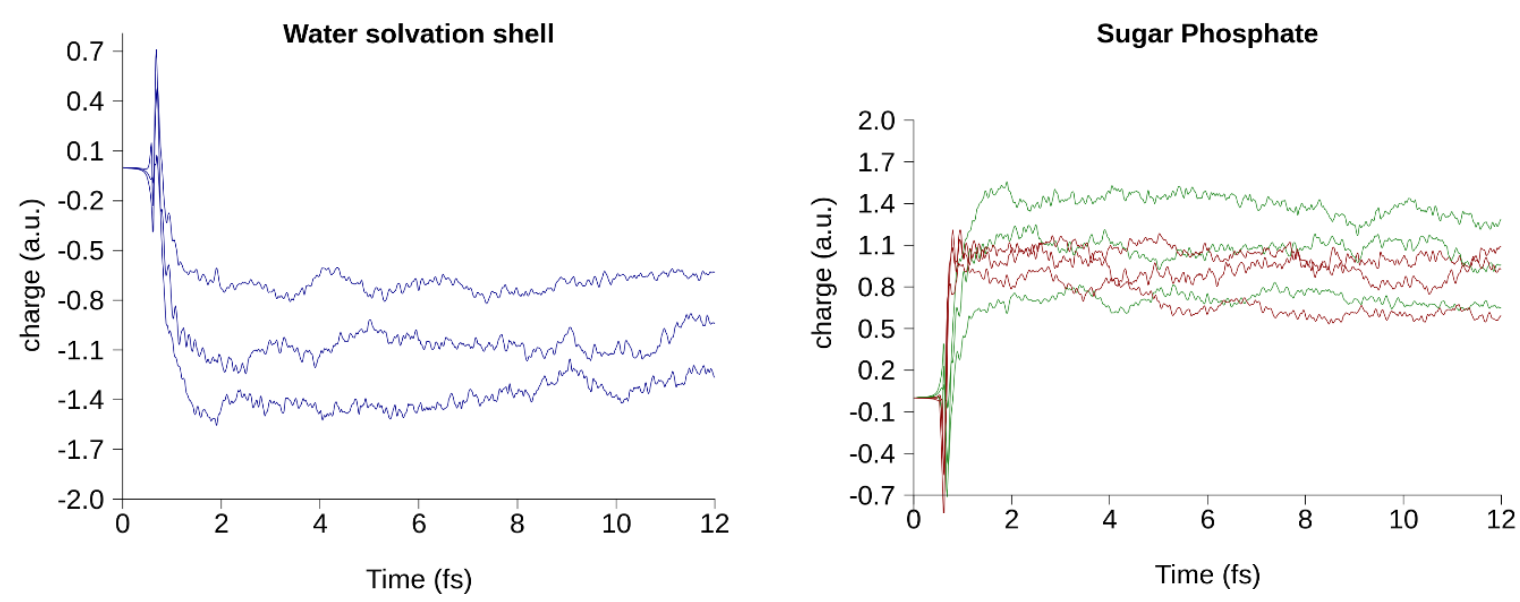

Figure 6: charge variation with respect to the ground state of the water shell (left), of the sugar moieties (right, red) and of the phosphate group $\left(\mathrm{O}_{3} \mathrm{PCH}_{2^{-}}\right.$, right, green) upon irradiation of the QM region by a $0.5 \mathrm{MeV}$ alpha particle. Simulations carried out within a non-polarizable QM/MM scheme.

\section{Conclusion}

We have been interested in this article in the ionization of the hydrated sugar-phosphate moieties of a double strand DNA oligomer triggered by collision with $0.5 \mathrm{MeV} \mathrm{He}^{2+}$ ions. Our work was motivated by recent experimental evidences on the redox properties of the $\mathrm{H}_{2} \mathrm{O}^{+}$cation against nucleoside/tides[15]. As discussed in Introduction, accessing the reactive properties of this peculiar chemical entity is challenging $[5,6,9,13,15]$, in particular when $\mathrm{H}_{2} \mathrm{O}^{+}$is produced within the hydration shell of large biomolecules. We have reported electron dynamics simulations carried out in the framework of hybrid RT-TD-ADFT/MM schemes relying either on polarizable or on non-polarizable embedding schemes. Our methodology permits to simulate charge migrations in extended molecular biological systems in a realistic manner.

We have focused on the very first stage of radiolysis, that is the production of hole and subsequent charge migrations. We have found that electron dynamics taking place during collision and after a few femtoseconds is not sensitive to the inclusion of induction within the MM part. This result can be understood noting that changes in embedding energy upon irradiation are minor compared to the change of energy within the QM region. The electronic excitations caused by collisions with charged 
particles are more important. The holes created on the water molecules or on the phosphate-sugar groups remain at the sites where they have been initially created. A pure charge migration process doesn't seem to be responsible for the quasi-direct effect. We recall that the simulations presented therein have been obtained under the fixed nuclear position approximation and within the adiabatic approximation. Nuclear motion could impact charge migration processes [47] and open the gate toward larger charge transfer. The investigations carried out in this work would deserve to be compared lifting this constraint, i.e. by enabling nuclear relaxation on the femtosecond time scale. Such simulations will further enable investigating the competition between electron and proton transfer within the DNA hydration shell. Such work is underway in our laboratory and will be reported in due course.

\section{Acknowledgments}

We thank the French Agence Nationale pour la Recherche (Project RUBI; grant number ANR-19-CE290011-01) and to the Grand Équipement National de Calcul Intensif (GENCl; project number A0060706913) and to Compute Canada for providing us with generous computational resources.

\section{References}

1. Becker D, Adhikary A, Sevilla MD (2010) Mechanisms of Radiation-Induced DNA Damage: Direct Effects. In: Recent Trends in Radiation Chemistry. WORLD SCIENTIFIC, pp 509-542

2. Sonntag C von (2010) Radiation-Induced DNA Damage: Indirect Effects. In: Recent Trends in Radiation Chemistry. WORLD SCIENTIFIC, pp 543-562

3. Elahe Alizadeh, Thomas M. Orlando, Léon Sanche (2015) Biomolecular Damage Induced by Ionizing Radiation: The Direct and Indirect Effects of Low-Energy Electrons on DNA. Annual Review of Physical Chemistry 66:379-398 . https://doi.org/doi:10.1146/annurev-physchem-040513-103605

4. Nolin F, Michel J, Wortham L, Tchelidze P, Balossier G, Banchet V, Bobichon H, Lalun N, Terryn C, Ploton D (2013) Changes to cellular water and element content induced by nucleolar stress: 
investigation by a cryo-correlative nano-imaging approach. Cellular and Molecular Life Sciences 70:2383-2394 . https://doi.org/10.1007/s00018-013-1267-7

5. Gauduel Y, Pommeret S, Migus A, Antonetti A (1990) Some evidence of ultrafast H2O+-water molecule reaction in femtosecond photoionization of pure liquid water: Influence on geminate pair recombination dynamics. Chem Phys 149:1-10 . http://dx.doi.org/10.1016/0301-0104(90)80126-I

6. Loh Z-H, Doumy G, Arnold C, Kjellsson L, Southworth SH, Al Haddad A, Kumagai Y, Tu M-F, Ho PJ, March AM, Schaller RD, Bin Mohd Yusof MS, Debnath T, Simon M, Welsch R, Inhester L, Khalili K, Nanda K, Krylov Al, Moeller S, Coslovich G, Koralek J, Minitti MP, Schlotter WF, Rubensson J-E, Santra $R$, Young $L$ (2020) Observation of the fastest chemical processes in the radiolysis of water. Science 367:179 . https://doi.org/10.1126/science.aaz4740

7. Marsalek O, Elles CG, Pieniazek PA, Pluhařová E, VandeVondele J, Bradforth SE, Jungwirth P (2011) Chasing charge localization and chemical reactivity following photoionization in liquid water. J Chem Phys 135:224510 . https://doi.org/doi:http://dx.doi.org/10.1063/1.3664746

8. LaVere T, Becker D, Sevilla MD (1996) Yields of $\mathrm{OH} \cdot$ in gamma-irradiated DNA as a function of DNA hydration: Hole transfer in competition $\mathrm{OH} \cdot$ formation. Radiat Res 145:673

9. Swarts SG, Becker D, Sevilla MD, Wheeler KT (1996) Radiation-induced DNA damage as a function of hydration. II. Base damage from electron-loss centers. Radiat Res 145:304

10. Balcerzyk A, El Omar AK, Schmidhammer U, Pernot P, Mostafavi M (2012) Picosecond Pulse Radiolysis Study of Highly Concentrated Nitric Acid Solutions: Formation Mechanism of NO3• Radical. J Phys Chem A 116:7302-7307 . https://doi.org/10.1021/jp304429f

11. Ma J, Schmidhammer U, Pernot P, Mostafavi M (2014) Reactivity of the Strongest Oxidizing Species in Aqueous Solutions: The Short-Lived Radical Cation H2O•+. J Phys Chem Lett 5:258-261 . https://doi.org/10.1021/jz402411x 
12. El Omar AK, Schmidhammer U, Balcerzyk A, LaVerne J, Mostafavi M (2013) Spur Reactions Observed by Picosecond Pulse Radiolysis in Highly Concentrated Bromide Aqueous Solutions. J Phys Chem A 117:2287-2293 . https://doi.org/10.1021/jp312023r

13. Ma J, Wang F, Mostafavi M (2018) Ultrafast Chemistry of Water Radical Cation, $\mathrm{H} 2 \mathrm{O} \bullet+$, in Aqueous Solutions. Molecules 23: . https://doi.org/10.3390/molecules23020244

14. Ma J, Denisov SA, Marignier J-L, Pernot P, Adhikary A, Seki S, Mostafavi M (2018) Ultrafast Electron Attachment and Hole Transfer Following lonizing Radiation of Aqueous Uridine Monophosphate. J Phys Chem Lett 9:5105-5109 . https://doi.org/10.1021/acs.jpclett.8b02170

15. MA J, Denisov SA, Adhikary A, Mostafavi M (2019) Ultrafast Processes Occurring in Radiolysis of Highly Concentrated Solutions of Nucleosides/Tides. International Journal of Molecular Sciences 20: . https://doi.org/10.3390/ijms20194963

16. López-Tarifa P, Gaigeot M-P, Vuilleumier R, Tavernelli I, Alcamí M, Martín F, Hervé du Penhoat M-A, Politis M-F (2013) Ultrafast Damage Following Radiation-Induced Oxidation of Uracil in Aqueous Solution. Angewandte Chemie International Edition 52:3160-3163. https://doi.org/10.1002/anie.201208038

17. Lai Y, Tsai M-Y, Tian Z, Qin N, Yan C, Hung S-H, Chi Y, Jia X (2020) A new open-source GPUbased microscopic Monte Carlo simulation tool for the calculations of DNA damages caused by ionizing radiation - Part II: sensitivity and uncertainty analysis. Medical Physics 47:1971-1982 . https://doi.org/10.1002/mp.14036

18. Cederbaum LS, Zobeley J (1999) Ultrafast charge migration by electron correlation. Chemical Physics Letters 307:205-210 . https://doi.org/10.1016/S0009-2614(99)00508-4

19. Alexander IK, Lorenz SC (2014) Ultrafast correlation-driven electron dynamics. J Phys B: At, Mol Opt Phys 47:124002 
20. Marcus RA, Sutin N (1985) Electron transfers in chemistry and biology. Biochim Biophys Acta 811:265-322 . http://dx.doi.org/10.1016/0304-4173(85)90014-X

21. Wang F, Schmidhammer U, de La Lande A, Mostafavi M (2017) Ultra-fast charge migration competes with proton transfer in the early chemistry of $\mathrm{H}^{2} \mathrm{O}^{\circ}+$. Phys Chem Chem Phys 19:2894-2899 . https://doi.org/10.1039/C6CP07013B

22. Calvayrac F, Reinhard PG, Suraud E (1995) Nonlinear plasmon response in highly excited metallic clusters. Phys Rev B 52:R17056-R17059 . https://doi.org/10.1103/PhysRevB.52.R17056

23. Yabana K, Bertsch GF (1996) Time-dependent local-density approximation in real time. Phys Rev B 54:4484-4487 . https://doi.org/10.1103/PhysRevB.54.4484

24. Pérez A, Marchán I, Svozil D, Sponer J, Cheatham lii TE, Laughton CA, Orozco M (2007) Refinement of the AMBER Force Field for Nucleic Acids: Improving the Description of $\alpha / \gamma$ Conformers. Biophys J 92:3817-3829 . http://dx.doi.org/10.1529/biophysj.106.097782

25. Piotr C, François-Yves D, Yong D, Junmei W (2009) Polarization effects in molecular mechanical force fields. J Phys: Condens Matter 21:333102

26. A. M. Koster GG (2018) deMon2k

27. de la Lande A, Alvarez-Ibarra A, Hasnaoui K, Cailliez F, Wu X, Mineva T, Cuny J, Calaminici P, López-Sosa L, Geudtner G, Navizet I, Garcia Iriepa C, Salahub DR, Köster AM (2019) Molecular Simulations with in-deMon2k QM/MM, a Tutorial-Review. Molecules 24: . https://doi.org/10.3390/molecules24091653

28. Wu X, Teuler J-M, Cailliez F, Clavaguéra C, Salahub DR, de la Lande A (2017) Simulating Electron Dynamics in Polarizable Environments. J Chem Theory Comput 13:3985-4002 . https://doi.org/10.1021/acs.jctc. 7b00251 
29. Calaminici P, Alvarez-Ibarra A, Cruz-Olvera D, Domínguez-Soria V-D, Flores-Moreno R, Gamboa GU, Geudtner G, Goursot A, Mejía-Rodríguez D, Salahub DR, Zuniga-Gutierrez B, Köster A (2016) Auxiliary Density Functional Theory: From Molecules to Nanostructures. In: Leszczynski J (ed) Handbook of Computational Chemistry. Springer Netherlands, Dordrecht, pp 1-67

30. Alvarez-Ibarra A, Parise A, Hasnaoui K, de la Lande A (2020) The physical stage of radiolysis of solvated DNA by high-energy-transfer particles: insights from new first principles simulations. Phys Chem Chem Phys 22:7747-7758 . https://doi.org/10.1039/D0CP00165A

31. Case DA, Cheatham TE, Darden T, Gohlke H, Luo R, Merz KM, Onufriev A, Simmerling C, Wang B, Woods RJ (2005) The Amber biomolecular simulation programs. J Comput Chem 26:1668-1688 . https://doi.org/10.1002/jcc.20290

32. Case DA, Darden TA, Cheatham III, Simmerling CL, Wang J, Duke RE, Luo R, Crowley M, Walker RC, Zhang W, Merz KM, Wang B, Hayik S, Roitberg A, Seabra G, Kolossváry I, Wong KF, Paesani F, Vanicek J, Wu X, Brozell SR, Steinbrecher T, Gohlke H, Yang L, Tan C, Mongan J, Hornak V, Cui G, Mathews DH, Seetin MG, Sagui C, Babin V, Kollman PA (2008) Amber 10. University of California, San Francisco

33. Alvarez-Ibarra A, Köster AM (2015) A new mixed self-consistent field procedure. Mol Phys 113:3128-3140 . https://doi.org/10.1080/00268976.2015.1078009

34. Perdew JP, Burke K, Ernzerhof M (1996) Generalized Gradient Approximation Made Simple. Phys Rev Lett 77:3865-3868

35. Dunning TH (1989) Gaussian basis sets for use in correlated molecular calculations. I. The atoms boron through neon and hydrogen. J Chem Phys 90:1007-1023 . https://doi.org/doi:http://dx.doi.org/10.1063/1.456153 
36. Köster AM, Flores-Moreno R, Reveles JU (2004) Efficient and reliable numerical integration of exchange-correlation energies and potentials. J Chem Phys 121:681-690 . https://doi.org/doi:http://dx.doi.org/10.1063/1.1759323

37. Castro A, Marques MAL, Rubio A (2004) Propagators for the time-dependent Kohn-Sham equations. J Chem Phys 121:3425-3433 . https://doi.org/10.1063/1.1774980

38. Cheng C-L, Evans JS, Van Voorhis T (2006) Simulating molecular conductance using real-time density functional theory. Phys Rev B 74:155112

39. Blackford LS, Choi J, Cleary AJ, D'Azevedo EF, Demmel J, Dhillon IS, Dongarra J, Hammarling S, -Henry G, Petitet A, Stanley K, Walker DW, Whaley RC (1997) ScaLAPACK: A Linear Algebra Library for Message-Passing Computers

40. Becke AD (1988) A multicenter numerical integration scheme for polyatomic molecules. J Chem Phys 88:2547-2553 . https://doi.org/doi:http://dx.doi.org/10.1063/1.454033

41. Salahub D, Noskov S, Lev B, Zhang R, Ngo V, Goursot A, Calaminici P, Köster A, Alvarez-Ibarra A, Mejía-Rodríguez D, Řezáč J, Cailliez F, de la Lande A (2015) QM/MM Calculations with deMon2k. Molecules 20:4780

42. Thole BT (1981) Molecular polarizabilities calculated with a modified dipole interaction. Chem Phys 59:341-350 . http://dx.doi.org/10.1016/0301-0104(81)85176-2

43. van Duijnen PT, Swart M (1998) Molecular and Atomic Polarizabilities: Thole's Model Revisited. J Phys Chem A 102:2399-2407 . https://doi.org/10.1021/jp980221f

44. Hirshfeld FL (1977) Bonded-atom fragments for describing molecular charge densities. Theoret Chim Acta 44:129-138 . https://doi.org/10.1007/bf00549096

45. de la Lande A, Clavaguéra C, Köster A (2017) On the accuracy of population analyses based on fitted densities\#. Journal of Molecular Modeling 23:99 . https://doi.org/10.1007/s00894-017-3264-5 
46. R Core Team (2018) R: A Language and Environment for Statistical Computing. R Foundation for Statistical Computing, Vienna, Austria

47. Andrea Rozzi C, Maria Falke S, Spallanzani N, Rubio A, Molinari E, Brida D, Maiuri M, Cerullo G, Schramm H, Christoffers J, Lienau C (2013) Quantum coherence controls the charge separation in a prototypical artificial light-harvesting system. Nature Communications 4:1602 https://doi.org/10.1038/ncomms2603 